International Journal of Food Science, Nutrition and Dietetics (IJFS)

ISSN 2326-3350

\title{
Marine Alga Sargassum Horneri Component And Bone Homeostasis: Role In Osteoporosis Prevention
}

Yamaguchi $\mathrm{M}^{*}$

Division of Diabetes, Endocrinology and Metabolism, Department of Medicine, Baylor College of Medicine, Houston, TX 77030, USA.

\section{Abstract}

Bone homeostasis is maintained through a balance between osteoblastic bone formation and osteoclastic bone resorption. Aging induces bone loss due to decreased osteoblastic bone formation and increased osteoclastic bone resorption. Osteoporosis with its accompanying decrease in bone mass is widely recognized as a major public heath problem. Nutritional factors may play a role in the prevention of bone loss with aging. Among marine algae of Undaria pinnatifida, Sargassum horneri, Eisenia bicyclis, Cryptonemia scmitziana, Gelidium amansii, and Ulva pertusa Kjellman which were gathered seasonally, Sargassum horneri (S. horneri) was found to have a unique anabolic effect on bone components. S. horneri extract had a stimulatory effect on osteoblastic bone formation and an inhibitory effects on osteoclastic bone resorption in vitro, thereby increasing bone mass. The intake of $S$. horneri extract caused a preventive effect on bone loss in animal models for osteoporosis and in healthy human. S. horneri extract, which is a functional food, may be usefulness as an osteogenic factor in preventing osteoporosis in human subjects.

Key Words: S. horneri extract, Osteoblastic bone formation, Osteoclastic bone resorption, Osteoporosis

\section{*Corresponding Author:}

Masayoshi Yamaguchi

Division of Diabetes, Endocrinology and Metabolism,

Department of Medicine, Baylor College of Medicine,

One Baylor Plaza, Houston, TX 77030, USA.

Tel: 713-798-6686, Fax: 713-798-8764.

E-mail: yamamasa1155@yahoo.co.jp

Received: January 18, 2013

Accepted: January 28, 2013

Published: January 31, 2013

Citation: Yamaguchi M (2013) Marine Alga Sargassum orneri Component and Bone Homeostasis: Role in Osteoporosis Prevention. Int J Food Sci Nutr Diet. 2(1), 9-14. doi: http://dx.doi.org/10.19070/2326-3350130003

Copyright: Yamaguchi M ${ }^{\oplus}$ 2013. This is an open-access article distributed under the terms of the Creative Commons Attribution License, which permits unrestricted use, distribution and reproduction in any medium, provided the original author and source are credited.

\section{Introduction}

Bone is a dynamic tissue that preserves skeletal size, shape, and structural integrity and to regulate mineral homeostasis. Bone homeostasis is maintained through a balance between osteoblastic bone formation and osteoclastic bone resorption. Aging and numerous pathological processes induce decrease in bone formation and increase in bone resorption, leading to osteoporosis, a devastating bone disease [1]. Osteoporosis, which is induced with decrease in bone mass, is widely recognized as a major public health problem. The most dramatic expression of the disease is represented by fractures of the proximal femur for which the number increases as the population ages [2].

Nutritional factors may have the potential effect to prevent bone loss with increasing age. There is growing evidence that the supplementation of nutritional and food factors may have the preventive effect on bone loss that is induced in animal model of osteoporosis and in human subjects [3-6]. Functional factors in food and plants, which regulate on bone homeostasis, have been to be worthy of notice in maintaining of bone health and prevention of bone loss with increasing age [7-13].

The effect of marine algae on bone metabolism was not determined so far. Undaria pinnatifida, Sargassum horneri, Eisenia bicyclis, Cryptonemia scmitriana, Gelidium amansii, and Ulva pertusa Kjellman are applied as food materials. Among these marine algae, Sargassum horneri ( $S$. horneri) was found to have a unique anabolic effect on bone components [14]. S. horneri extract has been found to have a stimulatory effect on osteoblastic bone formation and an inhibitory effect on osteoclastic bone resorption in vitro, thereby increasing bone mass.

This review has been written to outline the recent advances that have been made concerning the anabolic effect of $S$. horneri extract on bone metabolism and will discuss the usefulness of this functional food factor in prevention of osteoporosis.

\section{Cellular regulation of bone homeostasis}

Bone homeostasis is skillfully regulated through the functions of osteoblasts and osteoclasts, which are major cells in bone tissues [16-20]. Osteoclasts, which develop from hematopoietic progenitors, are recruited to the site and excavate the calcified matrix. In the physiologic process of bone turnover, a resorptive stimulus firstly triggers recruitment of osteoclasts to a site on the bone surface. During the initiation phase of bone resorption, osteoblasts arising from local mesenchymal stem cells assemble at the bottom of the cavity and bone formation begins. After the resorbed lacunar pit is filled with new osteoid, osteoblasts become flatter and less active, with the final newly remodeled bone surface lined by flat lining cells. As bone formation progresses, 
some osteoblasts are entombed within the matrix as osteocytes but the majority dies by apoptosis. When mechanical forces are reduced, for example in weightlessness, osteocytes die by apoptosis. This event appears to act as a beacon for osteoclast recruitment and generation of a new basic multicellular unit, which in turn replaces the old bone containing dead osteocytes with new bone containing viable osteocytes. Remodeling of cancellous bone begins with the retraction of lining cells that cover the bone surface $[19,20]$.

Bone acts as major storage site for growth factors, which are produced by osteoblasts, diffuse into newly deposited osteoid and are stored in the bone matrix including isulin-like growth factors (IGF- I and II), transforming growth factor- $\beta 1$ (TGF- $\beta 1$ ), platelet-derived growth factor (PDGF), or bone morphologic proteins (BMPs) $[21,22]$. These bone-derived factors, which can be liberated during subsequent periods of bone resorption, act in an autocrine, paracrine, or delayed paracrine fashion in the local microenvironment of the bone surface.

This process of bone remodeling to make bone unique among organs and tissues, and it also adds so many levels of complexity, with respect to interactions along the remodeling sequence by systemic influences (hormones), stress action on trabecular and cortical systems (physical activity/weight bearing), growth factors and cytokines produced by the bone cells which act locally on their own cell types and on the other bone cell types, or factors that come from nearby cells present in the marrow tissues.

\section{S. horneri extract stimulates osteoblastic bone for- mation}

Marine algae S. horneri [Sargassum horneri (Turner) C. Agardh] was seasonally gathered from the coast at Shimoda (Shizuoka Prefecture, Japan) and Miyako (Iwate Prefecture, Japan), and it was freeze-dried and powered [14]. The gathered fresh marine algae were homogenized in distilled water and are centrifuged at 5500 $\mathrm{g}$ in a refrigerated centrifuge for $10 \mathrm{~min}$. The $5500 \mathrm{~g}$ supernatant fraction was pooled for freeze-drying. The powder of the watersolubilized extract extract was dissolved in ice-cold distilled water for use in the experiments. The water-solubilized extract from $S$. borneri was purified through the method of membrane fractionation to collect active component of various molecular weights.

The direct effect of $S$. horneri extract on bone formation and mineralization has been examined using MC3T3 preosteoblastic cells in vitro [15]. The preosteoblastic cells were differentiated into mineralizing osteoblasts in vitro in the presence or absence of $S$. horneri extract in mineralizing medium for 21 days and stained for calcium deposition with Alizarin Red-S. S. horneri extract $(10$ and $25 \mu \mathrm{g} / \mathrm{ml}$ ) was found to potently enhance mineralization in MC3T3 cells [15]. The $S$. horneri extract $(25-100 \mu \mathrm{g} / \mathrm{ml}$ of medium) did not have a significant effect on cell number of MC3T3-E1 cells with culture. $S$. horneri extract did not appear to mediate direct toxic effects on the cultures as cells were proliferated robustly over the culture period, and were still alive and visibly attached to the plate at the end of the experiment as compared with those of control group.

Bone morphogenic proteins (BMPs) such as BMP-2 are anabolic agents that signal through the Smad signaling pathway [22]. The effect of $S$. horneri extract on basal and BMP-2-induced Smad activation has been shown using a Smad 4-luciferase reporter responsive to all Smad species [15]. S. horneri extract did not have a direct effect on basal Smad activation. $S$. horneri extract $(50$ or $100 \mu \mathrm{g} / \mathrm{ml}$ ) significantly enhanced Smad-activation induced by BMP-2. Also, S. horneri extract $(25-100 \mu \mathrm{g} / \mathrm{ml})$ significantly enhanced Smad-activation induced by TGF- $\beta 1$ [15].

NF- $\square \mathrm{B}$ activation is a potent inhibitory to osteoblast differentiation, and TNF- $\alpha$-induced NF- $\square \mathrm{B}$ activation leads to Smad suppression in MC3T3 osteoblast precursors [17,18]. Whether $S$. horneri extract is able to prevent TNF- $\alpha$-induced $\mathrm{NF}-x \mathrm{~B}$ activation in MC3T3 osteoblast precursors was examined [15]. $S$. horneri extract $(25-100 \mu \mathrm{g} / \mathrm{ml}$ of medium) significantly prevented TNF- $\alpha$-induced $\mathrm{NF}-x \mathrm{~B}$ activation, although $S$. horneri extract did not have a direct effect on basal NF- $x \mathrm{~B}$ activation.

Thus, $S$. horneri extract has been shown to stimulate osteoblastic differentiation and mineralization in vitro. TGF- $\beta 1$ - and BMP2 -induced activation of Smad signaling respectively plays an important role in the early commitment and differentiation of osteoblasts [22]. S. horneri extract was found to enhance BMP-2- or TGF- $\beta 1$-induced Smad activation with the dose-dependency [15]. $S$. horneri extract-induced Smad enhancement may be an important to stimulate osteoblastic differentiation and mineralization.

NF- $\square$ B signaling has been shown to downregulate osteoblast differentiation $[23,24]$. One major mechanism appears to involve the intersection of NF- $\square \mathrm{B}$ with the Smad signaling pathway [23]. NF- $\square \mathrm{B}$ signaling in osteoblasts intersects and disrupts Smad signaling by promoting production of Smad7, an inhibitor of TGF- $\beta 1$ - and BMP-induced R-Smad activation [25]. TNF- $\alpha$ further antagonizes BMP signaling by upregulating Smad ubiquitination regulatory factor 1 (Smurf1), promoting proteasomal degradation of bone morphogenetic signaling proteins [26]. Multiple suppressors of NF- $\square \mathrm{B}$ activation are capable of rescuing the inhibitory effect of TNF- $\alpha$ on BMP-2 and/or TGF$\beta 11$-induced Smad activation [23]. S. horneri extract was found to suppress $\mathrm{TNF}-\alpha$-induced $\mathrm{NF}-\varkappa \mathrm{B}$ activation in preosteoblastic MC3T3-E1 cells. This finding provides a possible mechanism by which $S$. horneri extract stimulates osteoblastic bone formation.

The anabolic effect of $S$. horneri extract on bone tissues has been found. Rat femoral-metaphyseal tissues were cultured in a medium containing water-solubilized extract $(25$ and $50 \mu \mathrm{g} / \mathrm{ml}$ ) obtained from U. pinnatifida, S. horneri, E. bicyclis, or C. scmitziana in vitro [14]. The bone calcium content was significantly elevated in the presence of $S$. horneri extract ( 25 and $50 \mu \mathrm{g} / \mathrm{ml}$ ). No effect was seen in the extracts of other marine algae. In addition, water suspensions $(5 \%)$ of marine alga powder were orally administered once daily for 7 days. Bone calcium content was significantly increased after the adminostration of U. pinnatifida, S. horneri, E. bicyclis, or C. scmitziana [14]. Also, bone alkaline phosphatase activity, which is an enzyme for calcification[27,28], was significantly enhanced with the administration of $S$. horneri or $G$. amansii. Thus, $S$. horneri extract has a unique anabolic effect on bone calcification in vitro and in vivo. This was the first time finding. The effects of $S$. borneri extract in increasing calcium content, alkaline phosphatase activity, and deoxyribonucleic acid (DNA) content in the femoraldiaphyseal and -metaphyseal tissues in vitro was completely abolished in the presence of cycloheximide, an inhibitor of protein synthesis [29]. DNA may be an index of cell number in bone tissues $[16,21]$ The anabolic effect of $S$. horneri extract may be resulted from newly synthesized protein components.

\section{S. horneri extract inhibits osteoclastic bone re- sorption}


S. horneri extract has been shown to suppress osteoclastic bone resorption. To examine the effect of $S$. horneri extract on osteoclast formation, RAW264.7 osteoclast precursors were differentiated into mature osteoclasts by stimulation with RANKL in the presence or absence of $S$. horneri extract with the dose range of 5 to $100 \mu \mathrm{g} / \mathrm{ml}$ of medium [15]. The $S$. horneri extract had no effect on cell number of preosteoclasts (RAW267.4 cells) with culture. S. horneri extract $(100 \mu \mathrm{g} / \mathrm{ml})$ did not have a toxicity to the precursors suppressing their proliferation over 7 days of culture [15]. S. horneri extract $(25-100 \mu \mathrm{g} / \mathrm{ml})$ significantly suppressed osteoclast formation induced by RANKL [15].

The NF- $\square$ B signal transduction pathway is essential for the generation of osteoclasts $[17,18]$. The effect of $S$. horneri extract on NF- $\square$ B activation by RANKL in osteoclast precursors has been examined. RAW 264.7 cells were transfected with an NF- $\square$ B reporter and stimulated with RANKL to induce NF- $\square$ B activity in the presence or absence of $S$. horneri $\operatorname{extract}(5-100 \mu \mathrm{g} / \mathrm{ml})$ [15]. $S$. horneri extract did not have a significant effect on basal NF- $x \mathrm{~B}$ activity [15]. However, RANKL-induced increase in NF- $\square$ B activity was significantly blunted in the presence of $S$. horneri extract [15].

S. horneri extract with dose-dependency, which did not have cell toxicity, was found to have a suppressive effect on RANKLstimulated osteoclastogenesis [15]. RANKL, the key osteoclastogenic cytokine, is central to formation of osteoclasts, the cells that resorb bone, through NF- $\square \mathrm{B}$ signaling $[17,18]$. $S$. borneri extract suppressed RANKL-induced NF- $\square$ B activation in osteoclast precursor cells [15]. This finding coincided with the observation that $S$. horneri extract suppresses RANKLinduced osteoclastogenesis [15]. S. horneri extract may inhibit osteoclastogenesis through suppression of $\mathrm{NF}-x \mathrm{~B}$ activation.

The effect of $S$. horneri on mineralization in MC3T3-E1 cells with culture for 21 days was observed at $10 \mu \mathrm{g} / \mathrm{ml}$ [15]. However, the effects of $S$. horneri on Smad activity in MC3T3-E1 cells and on NF- $\square$ B activity in RAW 264.7 cells with culture for 24 hours were observed at $25 \mu \mathrm{g} / \mathrm{ml}$ [15]. The effects of $S$. horneri on osteoclastogenesis in RAW 264.7 cells with culture for 6 days were also revealed at $25 \mu \mathrm{g} / \mathrm{ml}$ [15]. The effect of $S$. horneri on osteoblastogenesis and osteoclastogenesis may be observed with lower doses in the culture for longer periods.

The effects of $S$. horneri extract on bone resorption using femoral tissues in vitro have been examined [30]. Parathyroid hormone (PTH) - and prostagrandine E2 (PGE2) are known to induce osteoclastic bone resorption [31-33]. PTH- and PGE2-induced decreases in bone calcium content were completely inhibited after culture with water-solublilized extracts $(10,25$, and $50 \mu \mathrm{g} / \mathrm{ml})$ [30]. Also, the extract completely inhibited the PTH- or PGE2induced increase in medium glucose consumption and latic acid production by bone tissues [30]. Moreover, S. horneri extracts blocked the PTH-induced increase in acid phosphatase activity in the diaphyseal and metaphyseal tissues[30]. These findings indicate that the water-solubilized extracts of $S$. horneri have a direct inhibitory effect on bone resorption in tissue culture in vitro.

S. horneri extract possesses a potent Smad activation and antiNF- $\square \mathrm{B}$ activity and may have promise for development into an antiosteoporotic material capable of promoting new bone formation while simultaneously reducing bone resorption. S. horneri extract may be usefulness as a tool in the prevention of osteolysis with various pathophysiologic states. The mechanism by which $S$. borneri stimulates bone mass is summarized in Fig. 1.

\section{Characterization of active component in S. horneri extract}

Figure 1: S. horneri regulates bone homeostasis. S. horneri component stimulates osteoblastic bone formation and suppresses osteoclastic bone resorption, thereby increasing bone mass. S. horneri component enhances bone growth factors (TGF- $\beta 1$ and BMP-2)-induced Smad activation in osteoblasts and suppresses TNF- $\alpha$ - and RANL-enhanced NF- $x$ B activation in preosteoclasts.

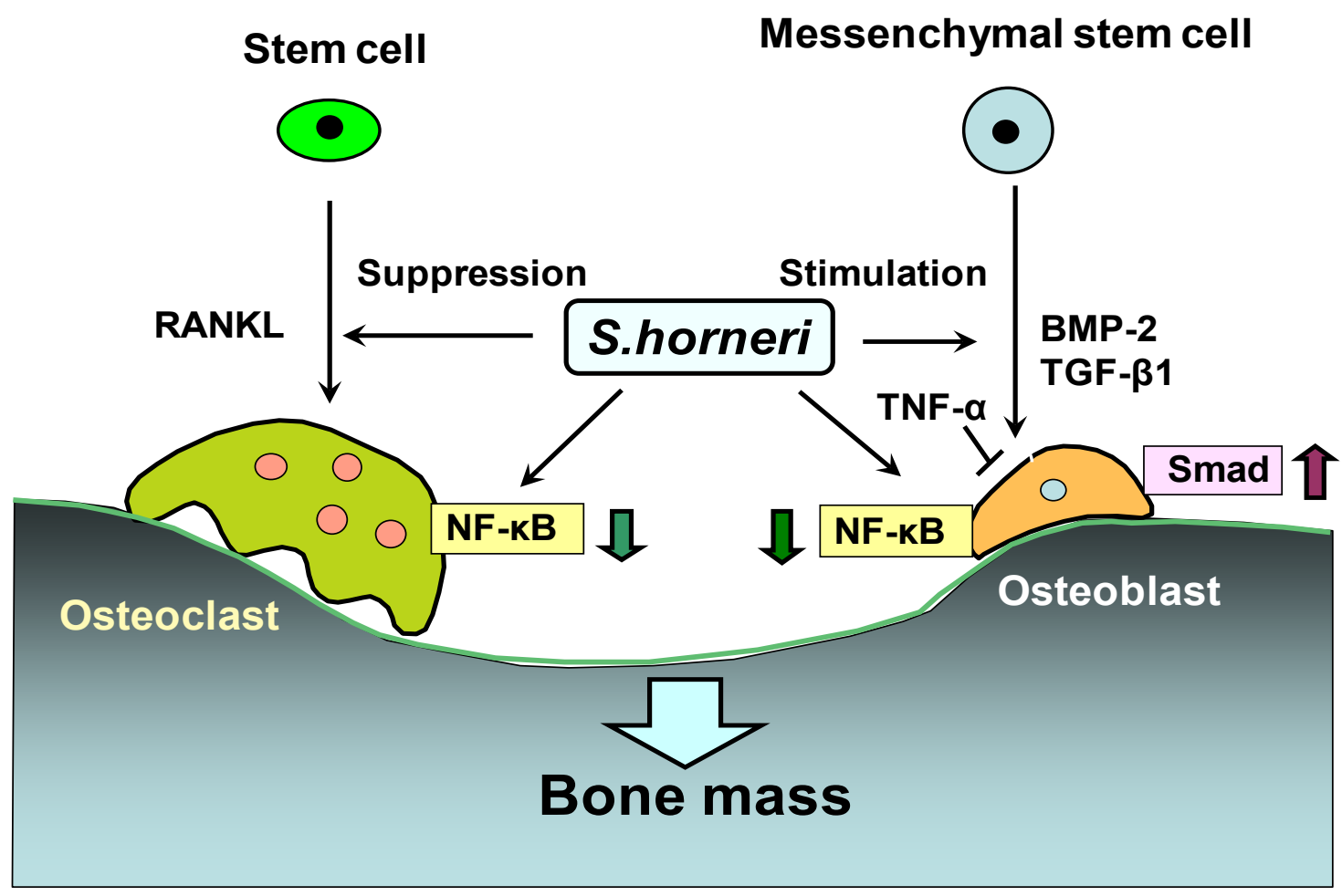


S. horneri extracts has been found to stimulate osteoblastic bone formation $[14,15,29]$ and to suppress osteoclastic bone resorption [30]. Those active components have been found to be present in S. horneri extract obtained from various coasts in both Japan (Shimoda and Iwate, Japan) and China [34]. The active component of $S$. horneri extract in stimulating bone calcification has been found to be near molecular weight (MW) 3000 [15,34]. Meanwhile, the active component of $S$. horneri extract in inhibiting osteoclastic cell formation was less than MW 3000 and over MW $50000[15,34]$. These components were stable under heat treatment. It has been speculated that the active component in stimulating bone calcification is a chemical but not peptide, and that the component over MW 50000 in inhibiting bone resorption may be a polysaccharide. These active components obtained from the coasts of Iwate (Japan) or China showed an identical molecular weight. The active component, which stimulates osteoblastogenesis and suppresses osteoclastogenesis, was found to be present in the components less than MW 3000 of S. horneri extract using preosteoblastic cells and RAW267.4 cells in vitro [15]. The active component that stimulates osteoblastic bone formation and suppresses osteoclastic bone resorption may be identical.

We found the existence of 4 chemicals in S. horneri components (less than MW 3000) using the analysis with liquid chromatography mass spectrophotometry system (LCMS-IT-TOF; Shimadzu, Kyoto, Japan). These chemicals were identified as 1,3,5-tris(oxolan2-ylmethyl)-1,3,5-triazinane (MW 339), 5-phenyl-2-[2-(5-phenyltetrazol-2-yl)ethyl]tetrazole (MW 318), 3-(hexadecylamino) propane-1,2-diol (MW 316), and 2-(2-hydroxyethyl-tridecyl-amino)ethanol (MW 288). These chemicals may have an effect on osteobalstogenesis and/or osteoclastogenesis, although their biological effects on bone cells remain to be elucidated. It is possible that combination of these compounds has an anabolic effect on bone.

\section{Anabolic effects of S. horneri extract on bone in vivo}

The anabolic effects of $S$. horneri extract on bone components in the femoral tissues of young and aged rats in vivo have been demonstrated [35]. Calcium content, alkaline phosphatase activity, and DNA content in the femoral-diaphyseal and -metaphyseal tissues of young male (4-week-old) rats were significantly increased after the administration of $S$. horneri extract $(25,50$, and $100 \mathrm{mg} / \mathrm{kg}$ ) for 7 days [35]. Moreover, these bone components in the femoral-diaphyseal and -metaphyseal tissues of aged female (50-week-old) rats were significantly increased after the administration of $S$. horneri extract $(100 \mathrm{mg} / \mathrm{kg})$ for 14 days, suggesting a preventive effect on bone loss with increasing age [35].

The intake of $S$. horneri extract has a preventive effect on bone loss in a pathophysiologic state has also been shown [36]. Diabetes has been shown to induce bone loss [37-39]. Streptozotocin (STZ) induces decrease in insulin secretion in pancreatic cells and causes type 1 diabetes. The oral administration of $S$. horneri extract (100 mg/ $\mathrm{kg}$ body weight) to STZ (60 mg/ $\mathrm{kg}$ body weight)-diabetic rats was found to have a preventive effect on bone loss with diabetes in vivo [36]. This finding suggests that the dietary intake of $S$. horneri extract has a preventive effect on bone loss in the pathophysiologic state. When the femoral tissues obtained from STZ-diabetic rats were cultured in medium containing $S$. horneri extract solution, the femoral calcium content and alkaline phosphatase activity were significantly increased in vitro[36]. Alkaline phosphatase is related to bone calcification $[27,28]$. S. horneri extract has a stimulatory effect on bone for- mation $[14,15,29]$ and an inhibitory effect on bone resorption in vitro $[15,30]$. Presumably, the preventive effect of $S$. horneri extract administration on diabetes-induced bone loss may be related to a direct action of the active component of $S$. horneri extract.

Interestingly, the oral administration of $S$. horneri extract (100 $\mathrm{mg} / \mathrm{kg}$ body weight) to STZ-diabetic rats has been found to have a significant preventive effect on the decrease in body weight and the increase in serum glucose and triglyceride levels induced in the diabetic state [36]. This was a novel finding. The intake of $S$. horneri extract has a partial restorative effect on serum biochemical finding with diabetes in vivo.

Thus, the intake of $S$. horneri extract has been demonstrated to have preventive effects on bone loss, hyperglycemia, and hyperlipidemia in STZ-diabetic rats [36]. The active component of $S$. borneri extract in preventing bone loss induced with diabetic state may be identical to the component that prevents an elevation of serum glucose and triglyceride levels with diabetes. This remains to be elucidated.

\section{Supplemental intake of S. horneri extract has an anabolic effect on bone metabolism in human subjects}

The supplemental intake of $S$. horneri extract has been shown to have an anabolic effect on bone metabolism in human subjects [40]. Study has been undertaken to determine the effect of supplemental intake of the water-solubilized $S$. horneri extract on circulating bone metabolic markers in healthy human [40]. Thirty-six volunteers, aged 20-60 years (16 men and 20 women), were enrolled. Volunteers were divided into three groups; placebo tablet without $S$. horneri extract ( 5 men and 7 women), tablet containing $S$. horneri extract at $300 \mathrm{mg} /$ day $(6 \mathrm{men}$ and 7 women) or $900 \mathrm{mg} /$ day (5 men and 6 women) [40]. Placebo or $S$. horneri extract tablet was ingested once a day for 4 or 8 weeks [40]. The intake of dietary $S$. horneri extract $(900 \mathrm{mg} /$ day) for 8 weeks did not have a significant alteration in other biochemical markers for the metabolic function of organs, suggesting that the intake does not have toxic effects in humans [40].

Bone-specific alkaline phosphatase [41] and $\gamma$-carboxylated osteocalcin [42] are serum bone markers of bone formation, and bone tartrate-resistant acid phosphatase (TRACP) [43] and Ntelopeptides of type I collagen [44] are markers of bone resorption. Serum bone-specific alkaline phosphatase or $\gamma$-carboxylated osteocalcin concentration was not significantly changed after the intake of $S$. horneri extract (300 or $900 \mathrm{mg} /$ day) for 4 or 8 weeks [40]. Serum bone tartrate-resistant acid phosphatase (TRACP) activity was significantly decreased after the intake of S. horneri extract (300 or $900 \mathrm{mg} /$ day) for 8 weeks [40]. Serum $\mathrm{N}$-telopeptides of type I collagen concentration was significantly decreased after the intake of $S$. horneri extract (900 mg/day) for 8 weeks [40]. Meanwhile, serum calcium, inorganic phosphorus, and other biochemical findings were not changed after the intake of $S$. horneri extract (300 or $900 \mathrm{mg} /$ day) for 4 or 8 weeks [40]. Thus, the prolonged intake of $S$. horneri extract has been found to have inhibitory effects on bone resorption in humans.

S. horneri extract has been shown to have a stimulatory effect on osteoblastic bone formation and an inhibitory effect on osteoclastic bone resorption. It has been speculated that the supplemental intake of $S$. horneri extract reveals suppressive effects on 
bone resorption, and later it exhibits stimulatory effects on bone formation in humans, thereby increasing bone mass. The supplemental intake of $S$. horneri extract may have a preventive effect on bone loss with increasing age and post-menopausal women.

\section{Conclusion}

Aging and numerous pathological processes induce decrease in bone formation and increase in bone resorption, leading to osteoporosis, which is induced with decrease in bone mass. Osteoporosis is widely recognized as a major public health problem. Functional food factors may have the potential effect to prevent bone loss with increasing age. There is growing evidence that the supplementation of food factors may have the preventive effect on bone loss that is induced in animal model of osteoporosis and in human subjects. Among various marine algae which are applied to food, $S$. horneri extract has been found to have a unique anabolic effect on bone mass using stimulating osteoblastic bone formation and depressing osteoclastic bone resorption. The intake of $S$. horneri extract has usefulness in prevention of bone loss with increasing age and pathologic state. This biomedical food material may play a role in maintaining of bone health and preventing of osteoporosis.

\section{Author Contribution}

Masayoshi Yamaguchi contributed to the design and conduct of the study, collection, analysis, and interpretation of data, and manuscript writing.

\section{Acknowledgement}

S. horneri extract was supplied through Masaaki Yokoyama and Toru Matsumoto, Biomaterial Department, Maruhachi Muramatsu, Inc., Yaizu, Japan.

\section{References}

[1]. Weitzmann MN, Pacifici R (2006) Estrogen deficiency and bone loss: an inflammatory tale. J Clin Invest 116: 1186-1194

[2]. Johnell O, Kanis JA (2006) An estimate of the worldwide prevalence and disability associated with osteoporotic fractures. Osteoporos Int 17: 1726-1733

[3]. Bonjour J-P, Schurch M-A, Rizzori R (1996) Nutritional aspects of hip fracture. Bone 18: 1395-1445

[4]. Yamaguchi M (2006) Regulatory mechanism of food factors in bone metabolism and prevention of osteoporosis. Yakugaku Zasshi 126:1117-1137

[5]. Yamaguchi M (2010) Nutritional Factors and Osteoporosis Prevention. Nova Science Publishers, Inc., New York

[6]. Yamaguchi M (2010) Role of nutritional zinc in the prevention of osteoporosis. Mol Cell Biochem 338: 241-254

[7]. Yamaguchi M (2002) Isoflavone and bone metabolism: Its cellular mechanism and preventive role in bone loss. J Health Sci 48: 209-222

[8]. YamaguchiM (2012) Nutritional factors and bone homeostasis: Synergistic effect of zinc and genistein osteogenesis. Mol Cell Biochem 366:201-221

[9]. Yamaguchi M (2012) Role of beta-cryptoxanthin in bone homeostasis. J Biomedical Sci, 19:36-48

[10]. Yamaguchi M, Hamamoto R, Uchiyama S, Ishiyama K (2008) Effects of flavonoid on calcium content in femoral tissue culture and parathyroid hormone-stimulated osteoclastogenesis in bone marrow culture in vitro. $\mathrm{Mol}$ Cell Biochem 303: 83-88

[11]. Yamaguchi M, Uchiyama S, Nakagawa T (2007) Preventive effects of bee pollen Cistus ladaniferus extract on bone loss in ovariectomiaed rats in vivo. J Health Sci 53: 571-575

[12]. Yamaguchi M, Ma ZJ, Suzuki T (2003) Anabolic effect of wasabi leafstalk (Wasabia Japonica MATSUM.) extract on bone components in the femoraldiaphyseal and -metaphyseal tissues of aged female rats in vitro and in vivo. J Health Sci 49: 123-128

[13]. Yamaguchi M (2012) Bone anabolic effect of wasabi leafstalk components: its related phytochemical p-hydroxycinnamic acid prevents osteoporosis. Open Access Scientific Reports 1:194. doi.10.4172/scientificreports.19

[14]. Yamaguchi M, Hachiya S, Hiratsuka S, Suzuki T (2001) Effect of marine algae extract on bone calcification in the femoral-metaphyseal tissues of rats: Anabolic effect of Sargassum horneri. J Health Sci 47: 533-538

[15]. Yamaguchi M, Matsumoto T (2012) Marine algae Sargassum horneri bioactive factor stimulates osteoblastogenesis and suppresses osteoclastogenesis in vitro. OA Biotechnology 1:3

[16]. Parfitt AM (1990) Bone-forming cells in clinical conditions. In: Hall BK, editor. Bone Volume 1. The Osteoblast and Osteocyte. Boca Raton, FL: Telford Press and CRC Press, pp351-429

[17]. Zaidi M, Blair HC, Moonga BS, Abe E, Huang C L-H (2003) Osteoclastogenesis, bone resorption, and osteoblast-based therapeutics. J Bone Miner Res 18: 599-609

[18]. Chambers TJ, Fuller K (2011) How are osteoclasts induced to resorb bone? Ann N Y Acad Sci 1240:1-6

[19]. Baron R, Vignery A, Horowitz M (1984) Lymphocytes, macrophages and the regulation of bone remodeling. Bone Miner Res 2:175-243

[20]. Raggatt LJ, Partridge C (2010) Cellular and molecular mechanisms of bone remodeling. J Biol Chem 285:25103-25108

[21]. Canalis E, McCarthy T, Centrella M (1988) Growth factors and the regulation of bone remodeling. J Clin Invest 81: 277-281

[22]. Chen G, Deng C, Li YP (2012) TGF- $\bigotimes$ and BMP signaling in osteoblast differentiation and bone formation. Int J Biol Sci 8:272-288

[23]. Li Y, Li A, Strait K, Zhang H, Nanes MS, Weitzmann MN (2007) Endogenous TNFalpha lowers maximum peak bone mass and inhibits osteoblastic Smad activation, through NF-kappaB. J Bone Miner Res 22: 646-655

[24]. Chang J, Wang Z, Tang E, Fan Z, McCauley L, Franceschi R, Guan K, Krebsbach PH, Wang CY (2009) Inhibition of osteoblastic bone formation by nuclear factor-kappaB. Nat Med 15:682-9

[25]. Eliseev RA, Schwarz EM, Zuscik MJ, O’Keefe RJ, Drissi H, Rosier RN (2006) Smad7 mediates inhibition of Saos 2 osteosarcoma cell differentiation by NFkappaB. Exp Cell Res 312:40-50

[26]. Guo R, Yamashita M, Zhang Q, Zhou Q, Chen D, Reynolds DG, Awad HA, Yanoso L, Zhao L, Schwarz EM, Zhang YE, Boyce BF, Xing L (2008) Ubiquitin ligase Smurf1 mediates tumor necrosis factor-induced systemic bone loss by promoting proteasomal degradation of bone morphogenetic signaling proteins. J Biol Chem 283:23084-92

[27]. Lian JB, Stein GS, Canalis E, Roby PG, Boskey AL (1999) Bone formation: Osteoblast lineage cells, growth factors, matrix proteins, and the mineralization process. In: Primer on the Metabolic Bone Diseases and Disorders of Mineral Metabolism (Favus, M. J., Eds.), 4th edition, Lippincott Williams \& Wilkins Press, New York pp 14-29

[28]. Yohay, DA, Zhang J, Thrailkill KM, Arthur JM, Quarles LD (1994) Role of serum in the developmental expression of alkaline phosphatase in MC3T3E1 osteoblasts. J Cell Physiol 158: 467-475

[29]. Uchiyama S, Yamaguchi M (2002) Stimulatory effect of Sargassum horner extract on bone formation in rat femoral-diaphyseal and -metaphyseal tissues in vitro. J Health Sci 48: 148-153

[30]. Uchiyama S, Yamaguchi M (2002) Inhibitory effect of marine alga Sargassum horneri extract on bone resorption in tissue culture in vitro. J Health Sci 48: $154-160$

[31]. Klein-Nulend J, Fall PM, Raisz LG (1990) Comparison of the effects of synthetic human parathyroid hormone (PTH)-(1-34)-related peptide of malignancy and bovine PTH-(1-34) on bone formation and resorption in organ culture. Endocrinology 126: 223-227

[32]. Graves L III, Jilka R L (1990) Comparison of bone and parathyroid hormone as stimulators of osteoclast development and activity in calvarial cell cultures from normal and osteopetrotic $(\mathrm{mi} / \mathrm{mi})$ mice. J Cell Physiol 145 : 102-109

[33]. Klein DC, Raisz LG (1970) Stimulation of bone resorption in tissue culture. Endocrinology 86: 1436-1440

[34]. Uchiyama, S, Hashizume, M., Hokari, Y., Nakagawa, T., Igarashi, A. \& Yamaguchi, M. (2004). Characterization of active component in marine alga Sargassum horneri extract in stimulating bone calcification in vitro. J Health Sci 50: 634-639

[35]. Uchiyama S, Yamaguchi M (2002) Anabolic effect of marine alga Sargassum horneri extract on bone components in the femoral-diaphyseal and -metaphyseal tissues of young and aged rats in vivo. J Health Sci., 48: 325-330

[36]. Uchiyama S, Yamaguchi M (2003) Preventive effect of marine alga Sargassum horneri extract on bone loss in streptozotocin-diabetic rats in vivo. J Health Sci 49: 149-155

[37]. Hui S L., Epstein S, Johanston C C (1985) A prospective study of bone mass in patients with type I diabetes. J Clin. Endocrinol Metab 60: 74-80

[38]. Mcnair P (1988) Bone mineral metabolism in human type I diabetes mellitus. Dan Med Bull 35: 109-121

[39]. Leslie WD, Rubin MR, Schwartz AV, Kanis JA (2012) Type 2 diabetes and bone. J Bone Miner Res 27:2231-2237

[40]. Matsumoto T, Hokari Y, Hashizume M, Yamaguchi M (2008) Effect of Sargassum horneri extract on circulating bone metabolic markers: Supplemental intake has an effect in health humans. J Health Sci 54: 50-55

[41]. Levy JR, Murray E, Manolagass S, Olefsky JM (1986) Demonstration of insulin receptors and modulation of alkaline phosphatase activity by insulin 
in rat osteoblastic cells. Endocrinology 119: 1786-1792

[42]. Price PA (1985) Vitamin K-dependent formation of bone gla protein (osteocalcin) and its function. Vitam Horm 42: 65-108

[43]. Hallen JM, Alatalo SL, Suminen H, Cheng S, Janekila A., \&Vaananen, H K (2000) Tartrate-resistant acid phosphatase 5b: A novel serum marker of bone resorption. J Bone Miner Res 15: 1337-1345

[44]. Clements JD, Herrick MV, Singer FR, Eyre DR (1997) Evidence that serum $\mathrm{NTx}$ (collagen-type I N-telopeptides) can act as an immunochemical marker of bone resorption. Clin Chem 43: 2058-2063 